\title{
MRI as a screening tool for prostate cancer: current evidence and future challenges
}

\author{
Christoph Würnschimmel ${ }^{1,2}$ - Thenappan Chandrasekar ${ }^{3}$. Luisa Hahn ${ }^{1} \cdot$ Tarik Esen $^{4}$. Shahrokh F. Shariat ${ }^{5,6,7,8,9}$. \\ Derya Tilki ${ }^{1,4,10}$
}

Received: 1 December 2021 / Accepted: 19 January 2022

(c) The Author(s) 2022

\begin{abstract}
Purpose Prostate cancer (PCa) screening, which relies on prostate-specific antigen (PSA) testing, is a contentious topic that received negative attention due to the low sensitivity and specificity of PSA to detect clinically significant PCa. In this context, due to the higher sensitivity and specificity of magnetic resonance imaging (MRI), several trials investigate the feasibility of "MRI-only" screening approaches, and question if PSA testing may be replaced within prostate cancer screening programs. Methods This narrative review discusses the current literature and the outlook on the potential of MRI-based PCa screening. Results Several prospective randomized population-based trials are ongoing. Preliminary study results appear to favor the "MRI-only" approach. However, MRI-based PCa screening programs face a variety of obstacles that have yet to be fully addressed. These include the increased cost of MRI, lack of broad availability, differences in MRI acquisition and interpretation protocols, and lack of long-term impact on cancer-specific mortality. Partly, these issues are being addressed by shorter and simpler MRI approaches (5-20 min bi-parametric MRI), novel quality indicators (PI-QUAL) and the implementation of radiomics (deep learning, machine learning).

Conclusion Although promising preliminary results were reported, MRI-based PCa screening still lack long-term data on crucial endpoints such as the impact of MRI screening on mortality. Furthermore, the issues of availability, cost-effectiveness, and differences in MRI acquisition and interpretation still need to be addressed.
\end{abstract}

Keywords Magnetic resonance imaging $\cdot$ MRI $\cdot$ Prostate cancer screening $\cdot$ Prostagram

\section{Introduction}

Prostate cancer $(\mathrm{PCa})$ detection has historically relied primarily on prostate-specific antigen (PSA) testing [1]. Suspicious PSA values and/or suspicious digital rectal

Derya Tilki

d.tilki@uke.de

1 Martini-Klinik Prostate Cancer Center, University Hospital Hamburg-Eppendorf, Martinistraße 52, 20246 Hamburg, Germany

2 Department of Urology, Lucerne Cantonal Hospital, Lucerne, Switzerland

3 Department of Urology, Thomas Jefferson University, Philadelphia, USA

4 Department of Urology, Koc University Hospital, Istanbul, Turkey

5 Department of Urology, Comprehensive Cancer Center, Medical University of Vienna, Vienna, Austria examination would trigger prostate biopsy ( $\mathrm{PBx})$. However, the "PSA pathway" is criticized for its weak sensitivity and specificity for clinically significant PCa diagnosis, which may result in over-diagnosis, and therefore overtreatment, of clinically insignificant $\mathrm{PCa}[2,3]$. For this reason, the

6 Department of Urology, Weill Cornell Medical College, New York, NY, USA

7 Department of Urology, University of Texas Southwestern, Dallas, TX, USA

8 Department of Urology, Second Faculty of Medicine, Charles University, Prague, Czech Republic

9 Institute for Urology and Reproductive Health, Sechenov University, Moscow, Russia

10 Department of Urology, University Hospital Hamburg-Eppendorf, Hamburg, Germany 
United States Preventive Services Task Force recommended against PCa screening relying on PSA in 2012; this was partly retracted in 2018, based on the established potential benefits of PSA screening with regard to lower PCa-specific mortality $[4,5]$. However, the true cancer-specific survival benefit of relying on PSA testing/screening is ambiguous and should be weighed against negative quality of life impact of PCa treatments. PSA screening should only be performed in well-informed men, taking into account patients' expectations, comorbidities and life expectancy [6]. Magnetic resonance imaging (MRI) of the prostate has evolved to be an integral part of the initial radiographic evaluation for patients at risk for PCa, together with PSA testing and digital rectal examination [7]. By identifying suspicious regions within the prostate and targeting these lesions on MRI-fusion guided PBx, detection rates of clinically significant $\mathrm{PCa}$ are increased, while detection rates of clinically insignificant $\mathrm{PCa}$ are reduced compared to a standard trans-rectal or trans-perineal ultrasound-guided PBx [8]. For this reason, recent European Association of Urology (EAU) guidelines recommend performing MRI prior to any $\mathrm{PBx}$ in men at elevated risk for PCa [6]. Therefore, the combination of PSA testing, digital rectal examination and prostate MRI represents current best practice in the evaluation of thoroughly informed men at risk for PCa. Furthermore, the EAU guidelines also incorporated established individual risk factors which should be taken into account when considering PCa diagnostics, such as positive family history, race/ethnicity and/or germline mutations such as BRCA2 [6]. With the additional diagnostic power of MRI, the over-diagnosis of clinically insignificant $\mathrm{PCa}$ and, subsequently, overtreatment could be reduced $[8,9]$.

While the combined "MRI-PSA-pathway" standard is now increasingly utilized, recent pilot studies have begun to question whether PSA testing can be replaced by MRI altogether for the purpose of PCa screening [10, 11]. It is hypothesized that this "MRI-only-pathway" reduces the need for invasive PBx without sacrificing the ability to detect clinically significant PCa (Table 1). Furthermore, it might be conceivable that the "MRI-only-pathway" may evolve to be the preferred method for PCa screening in future; this would resemble radiographic screening programs for other malignancies, such as the use of mammography for early detection of breast cancer. Currently, several trials evaluating the "MRI-only-pathway" are underway [12-14]. This narrative review aims to provide a contemporary overview on the current evidence while discussing potential obstacles encountered when relying on prostate MRI as a screening tool for PCa. The search strategy for this non-systematic literature review included combinations of keywords covering the general topic of PCa screening and included both PSA-based and MRI-based approaches. PubMed, Scopus, Web of Science, Google Scholar and abstract pages of the latest EAU and American Urological Association congresses as well as clinical trial registration websites (clinicaltrials. gov, isrctn.com) were used for literature retrieval. Reference lists of appropriate publications were screened for potential further relevant publications. Finally, relevant publications were discussed among authors for eligibility of inclusion within this review.

\section{Prostate cancer diagnosis with magnetic resonance imaging: technical considerations, image interpretation and pitfalls}

When performing an MRI for PCa diagnosis, bi-parametric (bpMRI) or multi-parametric MRI (mpMRI) is employed. For both bpMRI and mpMRI, T2-weighted images are critical for precise imaging of prostate anatomy. Diffusionweighted imaging (DWI), which assesses cell density,
Table 1 Prevalence of clinically significant prostate cancer (csPCa) according to prostate-specific antigen (PSA) values, derived from a secondary analysis of the Prostate, Lung, Colorectal, and Ovarian (PLCO) Cancer Screening Trial [40], as well as prevalence of csPCA according to PI-RADS scores 3-5, derived from the PRECISION trial [8] as well as the validation trial for the PI-RADS 2.0 system [41]

\begin{tabular}{lr}
\hline PSA value & csPCa prevalence \\
\hline$\leq 0.49-1.99 \mathrm{ng} / \mathrm{ml}$ & $0.3-4.0 \%$ \\
$2.0-3.99 \mathrm{ng} / \mathrm{ml}$ & $8.5-12.4 \%$ \\
$\geq 4.0 \mathrm{ng} / \mathrm{ml}$ & $23.1 \%$ \\
\hline PI-RADS score & csPCa prevalence \\
\hline 3 & $12.0-23.0 \%$ \\
4 & $49.0-60.0 \%$ \\
5 & $77.0-83.0 \%$ \\
\hline
\end{tabular}

csPCa was defined as any Gleason score $\geq 7$, death from PCa, or T2b status after radical prostatectomy in the PLCO trial and as any Gleason score $\geq 7$ in the PRECISION trial and the validation trial for PI-RADS 2.0 
further helps distinguish cancerous and non-cancerous regions, since a higher cell density is encountered in cancerous regions [15]. In the third step, which is excluded in bpMRI, dynamic contrast enhancement (DCE), which relies on contrast agents, more clearly delineates the vascular supply within the prostate, which is preferentially taken up by malignant PCa [16].

The most employed reporting standard for prostate MRI follows the European Society of Urogenital Radiology guidelines. These guidelines (Prostate Imaging Reporting \& Data System, PI-RADS), in their most recently updated and recommended PI-RADS version 2.1, assign a score to all concerning regions between grades one to five [17]. Based on this numerical grading, PI-RADS 1-2 are regarded as non-suspicious categories, PI-RADS 3 is regarded as "equivocal", and PI-RADS 4-5 are regarded as suspicious [18]. PI-RADS 4-5 lesions are often "biopsy triggers" in clinical practice, since they predict clinically significant $\mathrm{PCa}$ with a high degree of certainty $[18,19]$. On the other hand, PIRADS 3 lesions have been associated with a variable rate of concordance with clinically significant PCa. In addition, the prevalence of PI-RADS 3 was reported between 6 and 39\%, and some authors agree that the rate of PI-RADS 3 assignment by radiologists may serve as a surrogate parameter for understanding the MRI experience of the reader [20]. Excluding PI-RADS 1-2 on targeting can contribute to the reduction of unnecessary $\mathrm{PBx}[8,18]$.

MRI for detection of PCa has been extensively evaluated and MRI-based fusion biopsy significantly improves detection rates of clinically significant $\mathrm{PCa}$, compared to systematic biopsy alone [21-23]. For example, a recent randomized non-inferiority trial investigated the role of MRI-guided biopsies. Within this trial, Eklund et al. compared patients with a minimum PSA value of $3 \mathrm{ng} / \mathrm{ml}$ with one arm receiving a standard systematic biopsy and the other arm receiving a systematic biopsy and targeted biopsies of suspicious MRI findings. Eklund et al. reported a significantly higher rate of clinically significant PCa with MRI targeted biopsy, confirming the non-inferiority of the MRI-guided approach, despite lower rates of triggered biopsies in the experimental arm. [22]. Despite these important findings, the role of population-based PCa screening relying on MRI alone is underdeveloped and, therefore, not established. Some of the most important obstacles that still need to be addressed are the higher costs of mpMRI, limited availability and (time-consuming) expenditures of mpMRI, which is contradictory to the common principles of screening programs, which should be cost-effective, broadly available and most importantly, easy to apply and interpret [24-26]. To address cost-effectiveness, given the findings by Eklund et al., the combined benefit of detecting more clinically significant $\mathrm{PCa}$ while reducing rates of unnecessary biopsies using the MRI-based approach, the trade-off toward a potentially lower rate of overtreatment could hypothetically correspond to cost-savings [22]. Furthermore, some authors also advocate a simpler, faster and more cost-effective approach for prostate MRI, relying on bpMRI alone, which forgoes DCE and, therefore intravenous application of contrast agents, enabling faster acquisition times[27]. For example, in their meta-analysis of ten studies ( $n=1705$ patients) comparing bpMRI with mpMRI, Kang et al. could not detect statistically significant differences in PCa detection rates[28]. Furthermore, novel protocols with shorter acquisition times were also developed ("IMPROD") and further validated in a prospective multi-institutional setting [29, 30]. Using a 15-min bpMRI protocol, Jambor et al. reported of a sensitivity of 97\% (Confidence Interval [CI] 93-99\%) and a negative predictive value of $95 \%$ (CI 87-98\%) for the detection of Gleason Score $\geq 3+4$ in systematic \pm targeted biopsy. In another study by Weiss et al., acquisition times were even further lowered, down to $5 \mathrm{~min}$ [31]. Although this protocol was only tested on 52 patients within a single institution, the diagnostic accuracy was still reported to be comparable to standard mpMRI, which lays the foundation for a more feasible implementation of MRI within population-based PCa screening. Altogether, the potential of MRI as a potential screening tool still needs to be established, also in light of the higher demands and heterogeneity in interpreting MRI findings, that are far more challenging compared to relying on a simple PSA test alone.

\section{Future perspectives of magnetic resonance imaging for prostate cancer screening}

Currently, several ongoing large-scale population-based trials are evaluating MRI for PCa screening (Table 2). A Canadian trial (NCT02799303), entitled MVP (MRI vs. PSA) Trial, randomized 525 men into MRI-only versus PSA for PCa screening, for which results are pending publication. To-date, only preliminary details on recruitment were reported and are available as a conference abstract presented at the 2021 EAU meeting [32]. A Swedish trial (ISRCTN54449243), entitled "GÖTEBORG-2", which aims to recruit over 40,000 men, also investigates the benefit of MRI screening. However, this trial, with an estimated end date in 2040, relies on a combination of PSA and MRI screening [12], which therefore does not provide the strictest study endpoint, but might be more applicable in a broader population. In this regard, the ReIMAGINE trial, an MRI screening trial from a single institution in the United Kingdom (NCT04063566), uses an interesting primary outcome measure that includes acceptance rates of men for undergoing a 20-min MRI for screening purposes. Specifically, to assess this endpoint, men are randomly invited to take part in this trial at the level of consultations with general 


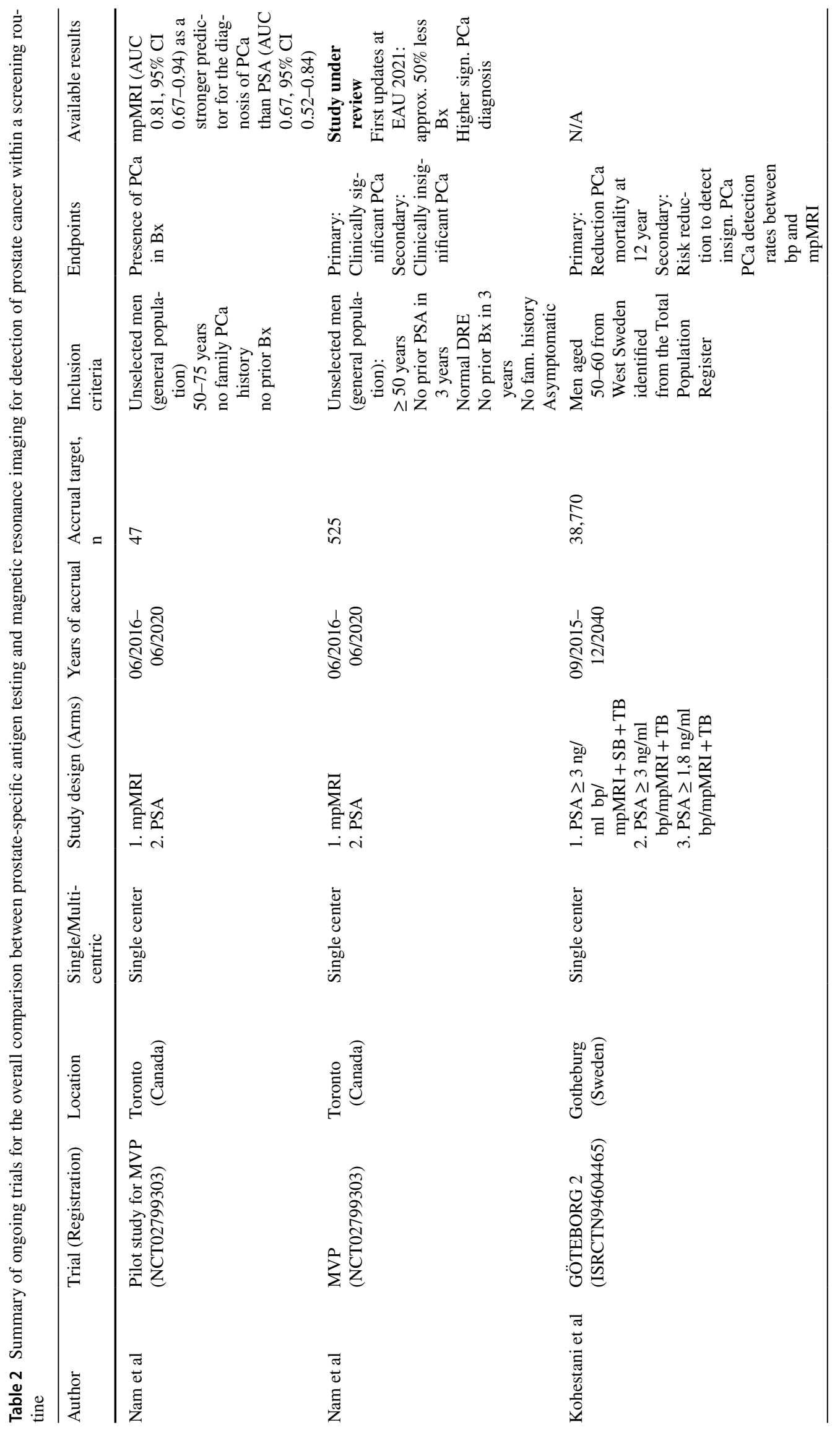




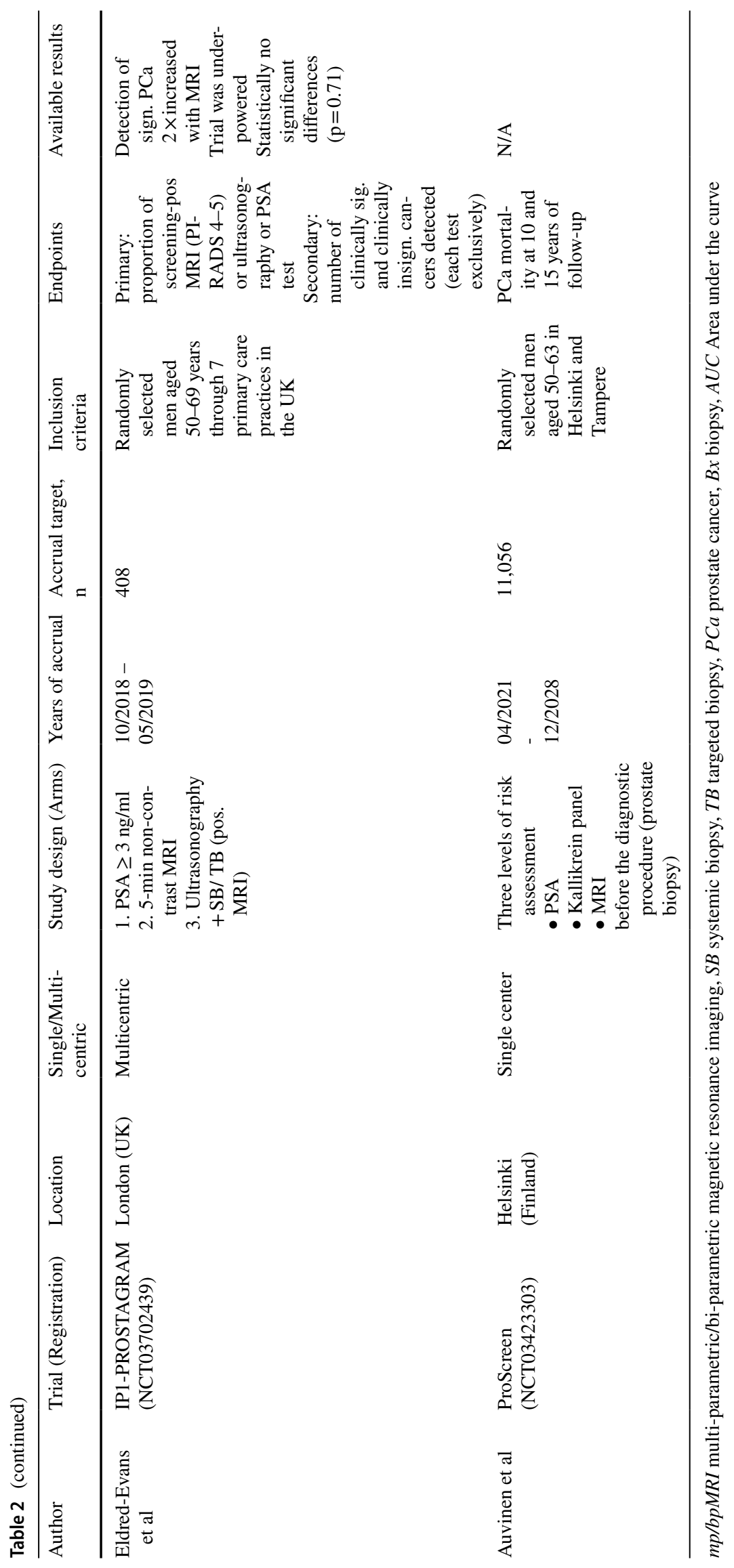


practitioners. Recently, ReIMAGINE reported an update stating that the recruitment goal had been reached $(n=309)$ and 303 men received a screening bpMRI and further results are being awaited [13]. All these above major trials will provide valuable information into the true prevalence of MRI lesions in a screening population and provide more insights with regard to the feasibility of MRI for population-based screening.

Apart from these ongoing trials, some data are already available. The IP1-PROSTAGRAM trial, a cohort study including data from 408 patients from seven primary care practices and two imaging centers in the United Kingdom (NCT03702439), recently reported favorable performance characteristics for bpMRI as a community-based screening test [14]. Specifically, the blinded IP1-PROSTAGRAM study defined cut-offs for a positive MRI (PIRADS 3-5) versus cutoffs for a positive PSA test ( $\geq 3 \mathrm{ng} / \mathrm{ml})$, either of which triggered a systematic PBx and additional fusion biopsy in the presence of suspicious lesions. In the comparison of positive PSA alone vs. positive MRI alone screening protocols, the latter was associated with higher rates of clinically significant $\mathrm{PCa}$, with no associated increase in the rate of clinically insignificant PCa. These early findings strengthen the role of MRI for PCa screening compared to PSA alone. However, cautious interpretation of these early findings is necessary. First, no further large-scale population-based data are available. Second, MRI screening has yet to demonstrate that higher rates of detecting clinically significant $\mathrm{PCa}$ results in a measurable and meaningful impact on $\mathrm{PCa}$-specific mortality compared to PSA screening programs or no screening at all. Therefore, the outcomes of the previously mentioned randomized trials, and further long-term observations of IP1-PROSTAGRAM, need to be awaited. Finally, the cost-effectiveness, availability and feasibility of MRI-based population screening remain issues that need to be resolved by health officials in future. Even if the issue of cost-effectiveness and availability are addressed, a major challenge for MRI screening remains providing a sufficient number of trained specialists for MRI acquisition and interpretation. Indeed, MRI acquisition and interpretation, despite the standardized PI-RADS v2.1 initiative, has a high rate of inter-observer variability and differences in common practice, especially in low-volume centers $[33,34]$. This appears especially relevant when considering the "MRI-only-pathway", since a misinterpretation of MRI findings due to lack of experience or incorrect MRI acquisition may potentially lead to under-detection and consequently under-treatment of clinically significant PCa. For this reason, from the current perspective, future pathways will most likely rely on the combination of both MRI and PSA pathways. Nevertheless, it is conceivable that the importance of MRI findings will increase compared to PSA testing within screening programs. Therefore, apart from the efforts to develop the necessary expertise to handle the growing demand of MRI acquisition and interpretation, novel and powerful artificial intelligence tools such as "machine learning/deep learning/ radiomics" might be a feasible supplementary approach for tackling this demand in future [35-37]. Indeed, comparative studies already provide first experiences with deep learning that provided similar results to conventional clinical MRI interpretation [38]. In addition, the different standards in MRI acquisition need to be addressed in future. One major step in this direction was recently done by the PRECISION study group that proposed a new quality scoring system for MRI acquisition (PI-QUAL) [39]. The authors defined PI-QUAL as a score on a Likert scale from 1 to 5. PI-QUAL 1 implies no sequences are of any diagnostic quality, while 5 implies that each sequence is independently of optimal diagnostic quality. Therefore, the combination of this new quality assessment tool, eventually combined with artificial intelligence systems and further funding and distribution of well-trained MRI personnel and advanced MRI technology, the possibility of MRIbased PCa screening may be technically feasible.

\section{Conclusion}

The implementation of MRI for prostate cancer diagnosis increased the diagnostic accuracy and helped reduce overdetection and overtreatment in clinical practice. Therefore, several trials are investigating the feasibility of "MRI-only" screening approaches, and assess if PSA testing could eventually be replaced within prostate cancer screening programs. Preliminary studies outline results that are in favor of the "MRI-only" approach. However, besides lack of long-term outcomes on crucial endpoints (such as the impact on cancerspecific mortality), further obstacles for implementing MRIbased screening programs (availability, cost-effectiveness, differences in MRI acquisition and interpretation) still need to be addressed.

Author contributions CW, DT take responsibility for the integrity of the review. CW, DT, TE, SFS: Project development, Manuscript writing/editing, TC, LH: critical review.

Funding Open Access funding enabled and organized by Projekt DEAL. None.

Availability of data and material Not applicable.

Code availability Not applicable.

\section{Declarations}

Conflict of interest Our research was conducted in the absence of any commercial or financial relationships that could be construed as a potential conflict of interest. The authors report no conflict of interest. 
Ethics approval This is a review article, ethical approval is therefore not relevant.

Human and animal rights Neither human participants nor animals were involved in this study.

Informed consent Informed consent is not relevant in a review article.

Open Access This article is licensed under a Creative Commons Attribution 4.0 International License, which permits use, sharing, adaptation, distribution and reproduction in any medium or format, as long as you give appropriate credit to the original author(s) and the source, provide a link to the Creative Commons licence, and indicate if changes were made. The images or other third party material in this article are included in the article's Creative Commons licence, unless indicated otherwise in a credit line to the material. If material is not included in the article's Creative Commons licence and your intended use is not permitted by statutory regulation or exceeds the permitted use, you will need to obtain permission directly from the copyright holder. To view a copy of this licence, visit http://creativecommons.org/licenses/by/4.0/.

\section{References}

1. Catalona WJ, Smith DS, Ratliff TL, Dodds KM, Coplen DE, Yuan JJJ et al (1991) Measurement of prostate-specific antigen in serum as a screening test for prostate cancer. $\mathrm{N}$ Engl $\mathrm{J}$ Med 324:1156-1161. https://doi.org/10.1056/NEJM199104253241702

2. Ilic D, Djulbegovic M, Jung JH, Hwang EC, Zhou Q, Cleves A et al (2018) Prostate cancer screening with prostate-specific antigen (PSA) test: a systematic review and meta-analysis. BMJ. https://doi.org/10.1136/bmj.k3519

3. Loeb S, Bjurlin MA, Nicholson J, Tammela TL, Penson DF, Carter HB et al (2014) Overdiagnosis and overtreatment of prostate cancer. Eur Urol 65:1046-1055. https://doi.org/10.1016/j. eururo.2013.12.062

4. Moyer VA (2012) Screening for prostate cancer: U.S. Preventive services task force recommendation statement. Ann Intern Med 157:120. https://doi.org/10.7326/0003-4819-157-2-20120 7170-00459

5. Grossman DC, Curry SJ, Owens DK, Bibbins-Domingo K, Caughey AB, Davidson KW et al (2018) Screening for prostate cancer. JAMA 319:1901. https://doi.org/10.1001/jama.2018.3710

6. Mottet N, van den Bergh RCN, Briers E, Cornford P, De Santis M, Fanti S et al (2020) EAU—ESTRO_ESUR—SIOG guidelines on prostate cancer 2020 European association of urology guidelines. European Association of Urology Guidelines Office, Arnhem

7. Rouvière $\mathrm{O}$, Puech $\mathrm{P}$, Renard-Penna R, Claudon $\mathrm{M}$, Roy C, Mège-Lechevallier F et al (2019) Use of prostate systematic and targeted biopsy on the basis of multiparametric MRI in biopsynaive patients (MRI-FIRST): a prospective, multicentre, paired diagnostic study. Lancet Oncol 20:100-109. https://doi.org/10. 1016/S1470-2045(18)30569-2

8. Kasivisvanathan V, Rannikko AS, Borghi M, Panebianco V, Mynderse LA, Vaarala MH et al (2018) MRI-targeted or standard biopsy for prostate-cancer diagnosis. N Engl J Med 378:17671777. https://doi.org/10.1056/NEJMoa1801993

9. Gandaglia G, Albers P, Abrahamsson P-A, Briganti A, Catto JWF, Chapple CR et al (2019) Structured population-based prostatespecific antigen screening for prostate cancer: the European association of urology position in 2019. Eur Urol 76:142-150. https:// doi.org/10.1016/j.eururo.2019.04.033
10. Grenabo Bergdahl A, Wilderäng U, Aus G, Carlsson S, Damber J-E, Frånlund $\mathrm{M}$ et al (2016) Role of magnetic resonance imaging in prostate cancer screening: a pilot study within the Göteborg randomised screening trial. Eur Urol 70:566-573. https://doi.org/ 10.1016/j.eururo.2015.12.006

11. Nam RK, Wallis CJD, Stojcic-Bendavid J, Milot L, Sherman C, Sugar L et al (2016) A pilot study to evaluate the role of magnetic resonance imaging for prostate cancer screening in the general population. J Urol 196:361-366. https://doi.org/10.1016/j.juro. 2016.01.114

12. Kohestani K, Månsson M, Arnsrud Godtman R, Stranne J, Wallström J, Carlsson S et al (2021) The GÖTEBORG prostate cancer screening 2 trial: a prospective, randomised, populationbased prostate cancer screening trial with prostate-specific antigen testing followed by magnetic resonance imaging of the prostate. Scand J Urol 55:116-124. https://doi.org/10.1080/21681805. 2021.1881612

13. Marsden T, McCartan N, Hadley J, Tuck S, Brown L, Haire AJ et al (2021) Update from the ReIMAGINE prostate cancer screening study NCT04063566: inviting men for prostate cancer screening using magnetic resonance imaging. Eur Urol Focus. https://doi.org/10.1016/j.euf.2021.03.027

14. Eldred-Evans D, Burak P, Connor MJ, Day E, Evans M, Fiorentino $\mathrm{F}$ et al (2021) Population-based prostate cancer screening with magnetic resonance imaging or ultrasonography. JAMA Oncol 7:395. https://doi.org/10.1001/jamaoncol.2020.7456

15. Giganti F, Pecoraro M, Fierro D, Campa R, Del Giudice F, Punwani S et al (2020) DWI and PRECISE criteria in men on active surveillance for prostate cancer: a multicentre preliminary experience of different ADC calculations. Magn Reson Imaging 67:50-58. https://doi.org/10.1016/j.mri.2019.12.007

16. Xu L, Zhang G, Shi B, Liu Y, Zou T, Yan W et al (2019) Comparison of biparametric and multiparametric MRI in the diagnosis of prostate cancer. Cancer Imaging 19:90. https://doi.org/ 10.1186/s40644-019-0274-9

17. Turkbey B, Rosenkrantz AB, Haider MA, Padhani AR, Villeirs G, Macura KJ et al (2019) Prostate imaging reporting and data system version 2.1: 2019 update of prostate imaging reporting and data system version 2. Eur Urol 2019(76):340-351. https:// doi.org/10.1016/j.eururo.2019.02.033

18. Israël B, van der Leest M, Sedelaar M, Padhani AR, Zámecnik P, Barentsz JO (2020) Multiparametric magnetic resonance imaging for the detection of clinically significant prostate cancer: what urologists need to know. Part 2: interpretation. Eur Urol 77:469-480. https://doi.org/10.1016/j.eururo.2019.10.024

19. Zhang L, Tang M, Chen S, Lei X, Zhang X, Huan Y (2017) A meta-analysis of use of prostate imaging reporting and data system version 2 (PI-RADS V2) with multiparametric MR imaging for the detection of prostate cancer. Eur Radiol 27:5204-5214. https://doi.org/10.1007/s00330-017-4843-7

20. Greer MD, Brown AM, Shih JH, Summers RM, Marko J, Law YM et al (2017) Accuracy and agreement of PIRADSv2 for prostate cancer mpMRI: a multireader study. J Magn Reson Imaging 45:579-585. https://doi.org/10.1002/jmri.25372

21. Mottet N, van den Bergh RCN, Briers E, Van den Broeck T, Cumberbatch MG, De Santis M et al (2021) EAU-EANMESTRO-ESUR-SIOG guidelines on prostate cancer-2020 update. Part 1: screening, diagnosis, and local treatment with curative intent. Eur Urol 79:243-262. https://doi.org/10.1016/j. eururo.2020.09.042

22. Eklund M, Jäderling F, Discacciati A, Bergman M, Annerstedt M, Aly $\mathrm{M}$ et al (2021) MRI-targeted or standard biopsy in prostate cancer screening. N Engl J Med. https://doi.org/10.1056/NEJMo a2100852

23. Goldberg H, Ahmad AE, Chandrasekar T, Klotz L, Emberton M, Haider MA et al (2020) Comparison of magnetic resonance 
imaging and transrectal ultrasound informed prostate biopsy for prostate cancer diagnosis in biopsy naïve men: a systematic review and meta-analysis. J Urol 203:1085-1093. https://doi.org/ 10.1097/JU.0000000000000595

24. Abreu AL (2020) The pillars for sustained growth of magnetic resonance imaging pathway for prostate cancer diagnosis: quality, reproducibility, accessibility, cost effectiveness, and training. Eur Urol 77:491-493. https://doi.org/10.1016/j.eururo.2020.01.004

25. Callender T, Emberton M, Morris S, Pharoah PDP, Pashayan N (2021) Benefit, harm, and cost-effectiveness associated with magnetic resonance imaging before biopsy in age-based and risk-stratified screening for prostate cancer. JAMA Netw Open 4:e2037657. https://doi.org/10.1001/jamanetworkopen.2020.37657

26. Hutchinson R, Lotan Y (2017) Cost consideration in utilization of multiparametric magnetic resonance imaging in prostate cancer. Transl Androl Urol 6:345-354. https://doi.org/10.21037/tau.2017. 01.13

27. Rais-Bahrami S, Siddiqui MM, Vourganti S, Turkbey B, Rastinehad AR, Stamatakis L et al (2015) Diagnostic value of biparametric magnetic resonance imaging (MRI) as an adjunct to prostatespecific antigen (PSA)-based detection of prostate cancer in men without prior biopsies. BJU Int 115:381-388. https://doi.org/10. 1111/bju.12639

28. Kang Z, Min X, Weinreb J, Li Q, Feng Z, Wang L (2019) Abbreviated biparametric versus standard multiparametric MRI for diagnosis of prostate cancer: a systematic review and meta-analysis. Am J Roentgenol 212:357-365. https://doi.org/10.2214/AJR.18. 20103

29. Jambor I, Boström PJ, Taimen P, Syvänen K, Kähkönen E, Kallajoki M et al (2017) Novel biparametric MRI and targeted biopsy improves risk stratification in men with a clinical suspicion of prostate cancer (IMPROD Trial). J Magn Reson Imaging 46:1089-1095. https://doi.org/10.1002/jmri.25641

30. Jambor I, Verho J, Ettala O, Knaapila J, Taimen P, Syvänen KT et al (2019) Validation of IMPROD biparametric MRI in men with clinically suspected prostate cancer: a prospective multiinstitutional trial. PLOS Med 16:e1002813. https://doi.org/10. 1371/journal.pmed.1002813

31. Weiss J, Martirosian P, Notohamiprodjo M, Kaufmann S, Othman $\mathrm{AE}$, Grosse U et al (2018) Implementation of a 5-minute magnetic resonance imaging screening protocol for prostate cancer in men with elevated prostate-specific antigen before biopsy. Invest Radiol 53:186-190. https://doi.org/10.1097/RLI.0000000000 000427

32. Nam RK (2021) Update on prostate cancer screning-session: riskadapted screening with MRI only: MVP trial (during the 2021 European Association of Urology, EAU 2021-Virtual Meeting, July $8-12,2021)$
33. Kohestani K, Wallström J, Dehlfors N, Sponga OM, Månsson M, Josefsson A et al (2019) Performance and inter-observer variability of prostate MRI (PI-RADS version 2) outside high-volume centres. Scand J Urol 53:304-311. https://doi.org/10.1080/21681 805.2019.1675757

34. Brembilla G, Dellglio P, Stabile A, Damascelli A, Brunetti L, Ravelli S et al (2020) Interreader variability in prostate MRI reporting using prostate imaging reporting and data system version 2.1. Eur Radiol 30:3383-3392. https://doi.org/10.1007/ s00330-019-06654-2

35. Chen JH, Asch SM (2017) Machine learning and prediction in medicine-beyond the peak of inflated expectations. N Engl J Med 376:2507-2509. https://doi.org/10.1056/NEJMp1702071

36. Aerts HJWL, Velazquez ER, Leijenaar RTH, Parmar C, Grossmann P, Carvalho S et al (2014) Decoding tumour phenotype by noninvasive imaging using a quantitative radiomics approach. Nat Commun 5:4006. https://doi.org/10.1038/ncomms5006

37. Bonekamp D, Schlemmer H-P (2021) Maschinelles Lernen und multiparametrische MRT in der Früherkennung des Prostatakarzinoms. Urologe 60:576-591. https://doi.org/10.1007/ s00120-021-01492-x

38. Schelb P, Kohl S, Radtke JP, Wiesenfarth M, Kickingereder P, Bickelhaupt S et al (2019) Classification of cancer at prostate MRI: deep learning versus clinical PI-RADS assessment. Radiology 293:607-617. https://doi.org/10.1148/radiol.2019190938

39. Giganti F, Allen C, Emberton M, Moore CM, Kasivisvanathan V (2020) Prostate imaging quality (PI-QUAL): a new quality control scoring system for multiparametric magnetic resonance imaging of the prostate from the PRECISION trial. Eur Urol Oncol 3:615619. https://doi.org/10.1016/j.euo.2020.06.007

40. Kovac E, Carlsson SV, Lilja H, Hugosson J, Kattan MW, Holmberg E et al (2020) Association of baseline prostate-specific antigen level with long-term diagnosis of clinically significant prostate cancer among patients aged 55 to 60 years: A Secondary Analysis of a Cohort in the Prostate, Lung, Colorectal, and Ovarian (PLCO) Cancer Screening Trial. JAMA Netw Open 3:e1919284. https://doi.org/10.1001/jamanetworkopen.2019. 19284

41. Hofbauer SL, Maxeiner A, Kittner B, Heckmann R, Reimann M, Wiemer L et al (2018) Validation of prostate imaging reporting and data system version 2 for the detection of prostate cancer. J Urol 200:767-773. https://doi.org/10.1016/j.juro.2018.05.003

Publisher's Note Springer Nature remains neutral with regard to jurisdictional claims in published maps and institutional affiliations. 